\title{
Late gadolinium enhancement imaging using spiral readouts at $3 \mathrm{~T}$
}

\author{
lain Pierce ${ }^{1,2^{*}}$, Jennifer Keegan ${ }^{1,2}$, Peter Drivas ${ }^{2}$, Peter D Gatehouse ${ }^{1,2}$, David N Firmin ${ }^{1,2}$ \\ From 16th Annual SCMR Scientific Sessions \\ San Francisco, CA, USA. 31 January - 3 February 2013
}

\section{Background}

Breath-hold late gadolinium enhancement (LGE) imaging is commonly performed as a stack of $2 \mathrm{D}$ short-axis slices through the ventricles. Standard inversion recovery with Cartesian k-space coverage requires long acquisition windows of 140 - $200 \mathrm{~ms}$ which have potential to introduce artefacts due to longitudinal magnetisation recovery and blurring due to cardiac motion. Ghosting in the phase encoding direction from beat-to-beat signal variations and due to poor breath-holding may mimic enhancement and a phase swapped (PS) stack of slices is also acquired to confirm diagnosis, although this doubles the scan time.

Spiral data acquisition provides highly efficient coverage of k-space and would potentially allow a significant

reduction of the acquisition window. This would reduce motion blurring and edge artefacts while at the same time, may eliminate the need for PS acquisitions. Its use for 2D LGE was investigated.

\section{Methods}

An interleaved spiral readout sequence was designed to match the current clinically-used standard inversionprepared segmented gradient-echo sequence in terms of spatial resolution $(1.4 \times 1.4 \times 6 \mathrm{~mm}$ (reconstructed $0.7 \mathrm{x}$ $0.7 \times 6 \mathrm{~mm})$ ) and breath-hold duration (14 cardiac cycles with alternate $\mathrm{R}$-wave gating). The sequence consisted of 12 interleaves (13 ms duration) with 2 acquired per cardiac cycle (flip angles $45^{\circ} \& 90^{\circ}$ ). The acquisition

\section{Cart $(/ / r)$}

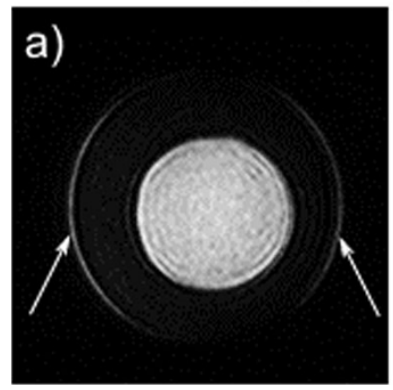

Cart (h/f)

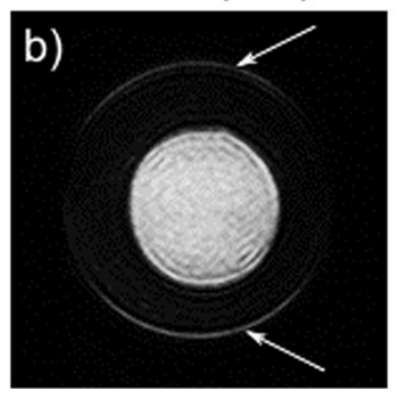

\section{Spiral}

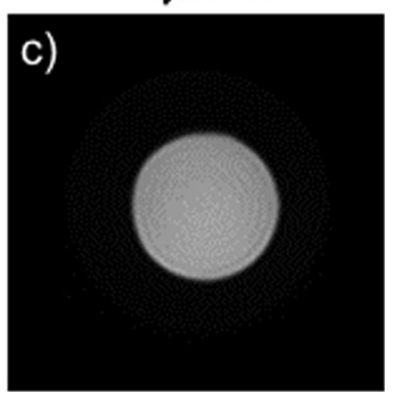

Figure 1 Images of a phantom using the standard LGE Cartesian sequence acquired using (a) left / right (l/r) and (b) head / foot (h/f) phase encoding and $(c)$ when using the spiral sequence. The phantom consists of two circular bottles with one placed inside the other, mimicking the left ventricular blood pool and myocardium. The bottles were filled with different concentrations of gadolinium such that the inner bottle (LV blood pool) had a shorter T1 than the outer bottle (myocardium). The Cartesian LGE images ((a) \& (b)) show edge artefact in the phase encoding direction, resulting from longitudinal magnetisation recovery through the long acquisition window (arrows). This artefact is not present on the LGE spiral image due to the much shorter acquisition window.

${ }^{1} \mathrm{NHLI}$, Imperial College London, London, UK

Full list of author information is available at the end of the article

(C) 2013 Pierce et al; licensee BioMed Central Ltd. This is an Open Access article distributed under the terms of the Creative Commons 
window was $53 \mathrm{~ms}$ compared to 144 - $204 \mathrm{~ms}$ for the standard sequence. A water excitation pulse eliminated signal from fat and associated off-resonance blurring.

Spiral LGE imaging was performed after the completion of clinical LGE studies (with and without PS) in 9 patients on a Siemens 3T Skyra scanner. Basal, mid and apical short axis slices were acquired for paired comparison with the standard Cartesian images in terms of blood signal-tonoise ratio (SNR) and blood/myocardium contrast-tonoise ratio (CNR).

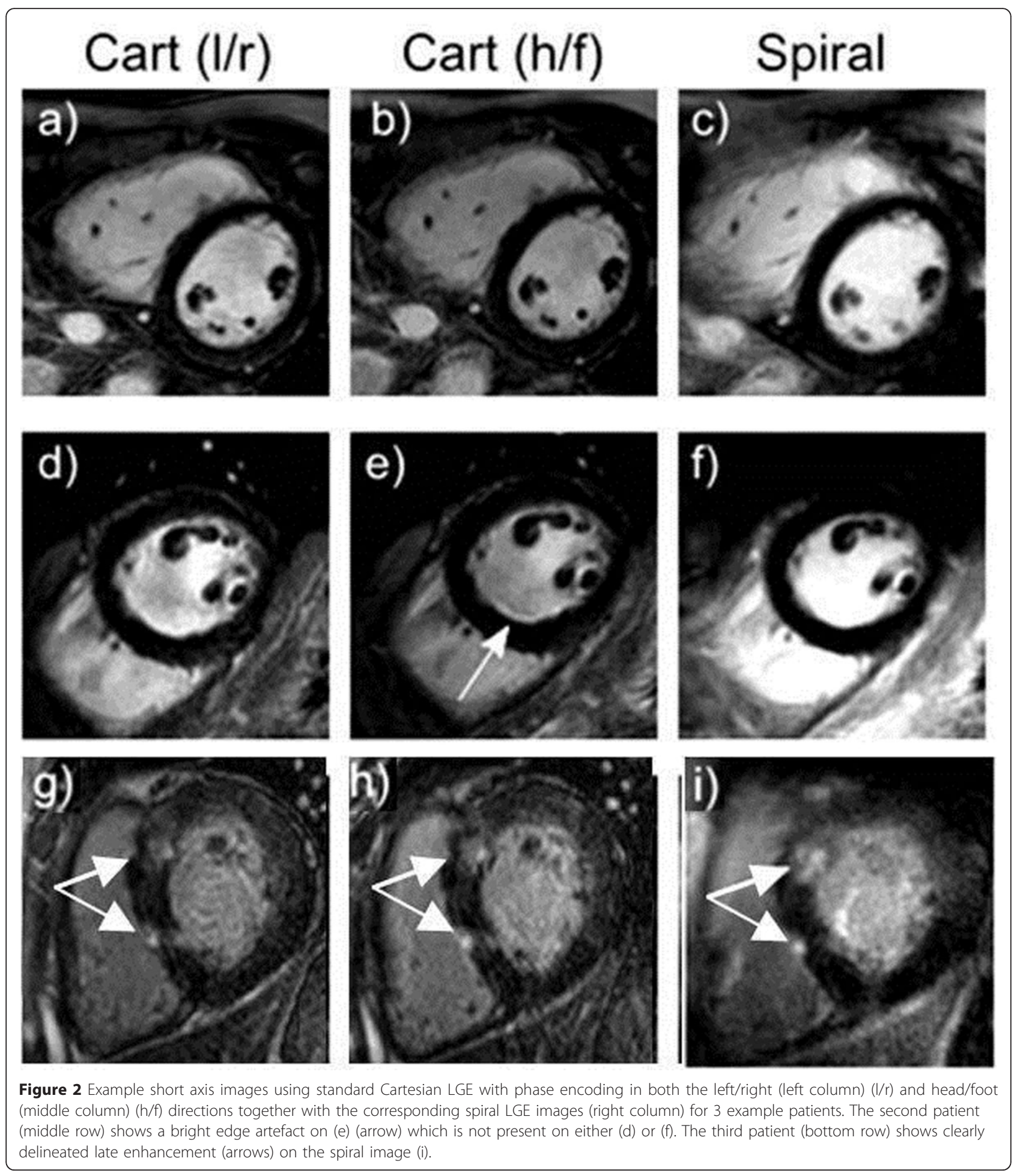




\section{Results}

Figure 1 shows phantom scans with edge artefact using Cartesian k-space coverage (due to magnetisation recovery through the long acquisition window) which are not present on the spiral image.

In vivo, blood pool SNR and blood-myocardium CNR were significantly higher with the spiral acquisitions (SNR: $162.8+/-62.5$ vs $238.8+/-112.0, \mathrm{p}<0.05$, CNR: $137.8+/-55.8$ vs $201.8+/-105.1, \mathrm{p}<0.05)$ despite always being acquired later when the blood pool gadolinium concentration is lower. Example images from both sequences in 3 patients are shown in Figure 2.

\section{Conclusions}

Spiral readouts allow shorter acquisition windows then standard Cartesian LGE imaging (53 ms compared to 144-204 $\mathrm{ms}$ in this study) which has the potential to reduce edge artefacts and motion blurring. The higher flip angles used also result in increased SNR and CNR.

While beat to beat signal variations result in ghosting in the phase encode direction with Cartesian acquisitions, requiring the acquisition of a PS stack, their effect with spiral acquisitions is less obvious and will be a focus of future work.

\section{Funding}

Wellcome Trust Grant WT093953MA

NIHR - National Institute for Health Research

\section{Author details}

${ }^{1} \mathrm{NHLI}$, Imperial College London, London, UK. ${ }^{2} \mathrm{NIHR}$ Royal Brompton

Cardiovascular Biomedical Research Unit, Royal Brompton Hospital,

London, UK

Published: 30 January 2013

doi:10.1186/1532-429X-15-S1-E8

Cite this article as: Pierce et al:: Late gadolinium enhancement imaging using spiral readouts at 3T. Journal of Cardiovascular Magnetic Resonance 2013 15(Suppl 1):E8.

\section{Submit your next manuscript to BioMed Central} and take full advantage of:

- Convenient online submission

- Thorough peer review

- No space constraints or color figure charges

- Immediate publication on acceptance

- Inclusion in PubMed, CAS, Scopus and Google Scholar

- Research which is freely available for redistribution

Submit your manuscript at www.biomedcentral.com/submit
C) Biomed Central 